\title{
Using Enhanced Russell Model to Solve Inverse Data Envelopment Analysis Problems
}

\author{
G. R. Jahanshahloo, F. Hosseinzadeh Lotfi, M. Rostamy-Malkhalifeh, and S. Ghobadi \\ Department of Mathematics, Islamic Azad University, Science and Research Branch, Tehran, Iran \\ Correspondence should be addressed to S. Ghobadi; ghobadi@iaukhsh.ac.ir
}

Received 23 February 2014; Accepted 7 April 2014; Published 5 May 2014

Academic Editors: A. Amirteimoori and M. Vaez-ghasemi

Copyright ( 2014 G. R. Jahanshahloo et al. This is an open access article distributed under the Creative Commons Attribution License, which permits unrestricted use, distribution, and reproduction in any medium, provided the original work is properly cited.

\begin{abstract}
This paper studies the inverse data envelopment analysis using the nonradial enhanced Russell model. Necessary and sufficient conditions for inputs/outputs determination are introduced based on Pareto solutions of multiple-objective linear programming. In addition, an approach is investigated to identify extra input/lack output in each of input/output components (maximum/minimum reduction/increase amounts in each a of input/output components). In addition, the following question is addressed: if among a group of DMUs, it is required to increase inputs and outputs to a particular unit and assume that the DMU maintains its current efficiency level with respect to other DMUs, how much should the inputs and outputs of the DMU increase? This question is discussed as inverse data envelopment analysis problems, and a technique is suggested to answer this question. Necessary and sufficient conditions are established by employing Pareto solutions of multiple-objective linear programming as well.
\end{abstract}

\section{Introduction}

Data envelopment analysis (DEA) is a nonparametric method based on linear programming for computing relative efficiencies of a decision making unit (DMU) by comparing it with other DMUs such that they produce the same multiple outputs by consuming the same multiple inputs. This method was first introduced by Charnes et al. (CCR model) [1] and extended by Banker et al. (BCC model) [2]. In the two recent decades, a wide range of research in operations research field has been allocated to this technique; see, for example, [3-5].

Relationships between DEA and MOLP can be applied as instruments in strategic planning and management control. These two types of models have much in common. However, DEA is to assess past performances as part of the management control function while MOLP is to plan future performances. These relationships have been used and developed by many scholars; see, for example, [6-12].

Inverse DEA is one of the most noticeable subjects both practically and theoretically. This concept first was discussed by Zhang and Cui [13]. Some of the questions are introduced by Wei et al. [14] in inverse DEA filed. They considered inverse DEA to answer this question: "if among a group of DMUs, we increase certain inputs to a particular unit and assume that the DMU maintains its current efficiency level with respect to other DMUs, how much should the outputs of the DMU increase?" In order to answer this question, Wei et al. [14] and Yan et al. [15] offered a linear programming problem to estimate the outputs when the unit under assessment is weakly efficient and a MOLP model when the unit under assessment is inefficient.

Moreover, in the following, Hadi-Vencheh et al. [16, 17] attempted to answer this question: "if among a group of DMUs, we increase certain outputs to a particular unit and assume that the DMU maintains its current efficiency level with respect to other DMUs, how much should the inputs of the DMU increase?" In their studies, the proposed models by Wei et al. [14] have been developed. After introducing inverse DEA, some of researchers studied it theoretically and practically [15-21].

In all investigations done on inverse DEA, researchers considered radial models such as CCR [1], BCC [2], ST [22], and FG [23] models. As it is known, the nonradial models have some different properties compared with the radial models. Therefore, in some cases, answering the questions presented in the literature inverse DEA with the 
nonradial models can provide more appropriate information. Consequently, for more suitable analysis, one of the nonradial models, for instance, additive models or slack-based models, can be considered. In this research, to solve some of the above problems, the nonradial Enhanced Russell Model (ERM) [24] is considered. In addition, a new problem in inverse DEA field is introduced: "if among a group of DMUs for a particular unit, the decision maker is required to increase inputs and outputs, in which, the DMU maintains its current efficiency level with respect to other DMUs, how much should the inputs and outputs of the DMU increase?" Pareto solutions of MOLP are used to solve this problem.

The paper is organized as follows. In Section 2, DEA models are reviewed and extended and the problem is stated in DEA terms. It is shown that how the inverse DEA problem (increment of the outputs) can be converted to and solved by a multiobjective programming problem, when the DMU is ERM efficient. If there exists a lack in each of the output components, its amount is specified. In Section 3, the question proposed by Wei et al. [14] is answered, when the DMU is ERM efficient. Likewise, if there exists an extra in each of the input components, its amount is specified. The new problem is solved in Section 4. For a special decision making unit providing that the ERM-efficiency score remains unchanged, necessary and sufficient conditions are introduced based on Pareto solutions of multiple-objective linear programming problems to find the minimum and maximum increase of input and output vectors, respectively. In Section 5, two examples are used to illustrate our calculation method. Finally, Section 6 demonstrates some conclusions and suggestions for future research.

\section{Estimate Inputs}

Let us consider a set of $\mathrm{DMU}_{s}\left\{\mathrm{DMU}_{j}: j=1, \ldots, n\right\}$, in which $\mathrm{DMU}_{j}$ produces multiple outputs $y_{r j}(r=1, \ldots, s)$, by utilizing multiple inputs $x_{i j}(i=1, \ldots, m)$. Let input and output for $\mathrm{DMU}_{j}$ be denoted by $X_{j}=\left(x_{1 j}, x_{2 j}, \ldots, x_{m j}\right)^{t}$ and $Y_{j}=\left(y_{1 j}, y_{2 j}, \ldots, y_{s j}\right)^{t}$, respectively. Also, suppose $X_{j} \supsetneqq 0$ and $Y_{j} \supsetneqq 0$. The nonradial enhanced Russell model (ERM) [24] is considered for measuring the relative efficiency of the unit under assessment $\mathrm{DMU}_{o}, o \in\{1,2, \ldots, n\}$, as follows:

$$
\begin{aligned}
\rho_{o}^{*}=\min \quad & \frac{(1 / m) \sum_{i=1}^{m} \theta_{i}}{(1 / s) \sum_{r=1}^{s} \varphi_{r}} \\
\text { s.t. } \quad & \sum_{j=1}^{n} \lambda_{j} x_{i j} \leq \theta_{i} x_{i o}, \quad i=1, \ldots, m \\
& \sum_{j=1}^{n} \lambda_{j} y_{r j} \geq \varphi_{r} y_{r o}, \quad r=1, \ldots, s \\
& \theta_{i} \leq 1, \quad \varphi_{r} \geq 1, \quad i=1, \ldots, m, r=1, \ldots, s, \\
& \lambda \in \Omega,
\end{aligned}
$$

where

$$
\begin{gathered}
\Omega=\left\{\lambda \mid \lambda=\left(\lambda_{1}, \ldots, \lambda_{n}\right), \delta_{1}\left(\sum_{j=1}^{n} \lambda_{j}+\delta_{2}(-1)^{\delta_{3}} v\right)=\delta_{1},\right. \\
\left.v \geq 0, \lambda_{j} \geq 0, j=1, \ldots, n\right\} .
\end{gathered}
$$

Here $\delta_{1}, \delta_{2}$, and $\delta_{3}$ are parameters with $0-1$ values. It is obvious that if $\delta_{1}=0$, then model (1) is under constant returns to scale (CRC), if $\delta_{1}=1$ and $\delta_{2}=0$, then model (1) is under variable returns to scale (VRS), if $\delta_{1}=\delta_{2}=1$ and $\delta_{3}=0$, then model (1) is under a nonincreasing returns to scale (NIRS), and if $\delta_{1}=\delta_{2}=\delta_{3}=1$, then model (1) is under a nondecreasing returns to scale (NDRS) assumption of the production technology.

Remark 1. Although in this study under discussion results satisfy CRC, VRS, NIRS, and NDRS assumption of the production technology, but the CRC model is considered only for simplicity.

Definition 2 (ERM-efficiency, see [24]). The optimal value $\rho_{o}^{*}$ of the model (1) is called the ERM-efficiency score of $\mathrm{DMU}_{o}$. $\mathrm{DMU}_{o}$ is ERM efficient, if and only if $\rho_{o}^{*}=1$ (this condition is equivalent to $\theta_{i}^{*}=1$ and $\varphi_{r}^{*}=1$ for each $i=1, \ldots, m$, $r=1, \ldots, s$ in any optimal solution).

In [13] Zhang and Cui introduced inverse DEA. Since then this problem has allocated to itself some of researches in DEA field; see, for example [5, 14, 16-19, 21]. Based upon investigated results by Hadi-Vencheh et al. [16], this question is considered: suppose that the $\mathrm{DMU}_{o}$ is ERM efficient, if the ERM-efficiency score of $\mathrm{DMU}_{o}$ remains unchanged, but the outputs increase, how much should the inputs of the $\mathrm{DMU}_{o}$ increase?

To answer the above question, until the end of this section, presume that the outputs of $\mathrm{DMU}_{o}$ are increased from $Y_{o}$ to $\beta_{o}=Y_{o}+\Delta Y_{o}$, where $\Delta Y_{o} \supsetneqq 0$. The objective of the problem is to estimate the input vector on the condition that $\mathrm{DMU}_{o}$ is still ERM efficient. In fact,

$$
\alpha_{o}^{*}=\left(\alpha_{1 o}^{*}, \alpha_{2 o}^{*}, \ldots, \alpha_{m o}^{*}\right)^{t}=X_{o}+\Delta X_{o}, \quad \Delta X_{o} ¥ 0 .
$$

Assume $\mathrm{DMU}_{n+1}$ represents $\mathrm{DMU}_{o}$ after modification of the inputs and outputs. The following model is considered to calculate the ERM efficiency of $\mathrm{DMU}_{n+1}$ :

$$
\begin{aligned}
\rho_{o}^{+*}=\min & \frac{(1 / m) \sum_{i=1}^{m} \theta_{i}}{(1 / s) \sum_{r=1}^{s} \varphi_{r}} \\
\text { s.t. } \quad & \sum_{j=1}^{n} \lambda_{j} x_{i j}+\alpha_{i o}^{*} \lambda_{n+1} \leq \theta_{i} \alpha_{i o}^{*}, \quad i=1, \ldots, m \\
& \sum_{j=1}^{n} \lambda_{j} y_{r j}+\beta_{r o} \lambda_{n+1} \geq \varphi_{r} \beta_{r o}, \quad r=1, \ldots, s
\end{aligned}
$$




$$
\begin{aligned}
& \theta_{i} \leq 1, \quad \varphi_{r} \geq 1, \quad i=1, \ldots, m, \quad r=1, \ldots, s \\
& \lambda_{j} \geq 0, \quad j=1, \ldots, n+1 .
\end{aligned}
$$

Along with [16], the following definition is considered.

Definition 3. If the optimal values of models (1) and (4) are equal, it is said that the ERM efficiency remains unchanged; that is, $\operatorname{eff}\left(\alpha_{o}^{*}, \beta_{o}\right)=\operatorname{eff}\left(X_{o}, Y_{o}\right)$.

To answer the above question, based on the results of [16], the following MOLP model is considered:

$$
\begin{array}{ll}
\min & \left(\alpha_{1 o}, \ldots, \alpha_{m o}\right) \\
\text { s.t. } & \sum_{j=1}^{n} \lambda_{j} x_{i j} \leq \theta_{i}^{*} \alpha_{i o}, \quad i=1, \ldots, m \\
& \sum_{j=1}^{n} \lambda_{j} y_{r j} \geq \varphi_{r}^{*} \beta_{r o}, \quad r=1, \ldots, s \\
& \alpha_{i o} \geq x_{i o}, \quad i=1, \ldots, m \\
& \lambda_{j} \geq 0, \quad j=1, \ldots, n,
\end{array}
$$

where $\left(\theta^{*}=\left(\theta_{1}^{*}, \ldots, \theta_{m}^{*}\right), \varphi^{*}=\left(\varphi_{1}^{*}, \ldots, \varphi_{s}^{*}\right)\right)$ is an optimal solution to problem (1).

Definition 4 (see [16]). Let $\left(\lambda^{*}, \alpha_{o}^{*}\right)$ be a feasible solution to problem (5). If there is no feasible solution $\left(\lambda, \alpha_{o}\right)$ of (5) such that $\alpha_{i o} \leq \alpha_{i o}^{*}$ for all $i=1, \ldots, m$ and $\alpha_{i o}<\alpha_{i o}^{*}$ for at least one $i$, then it is said the $\left(\lambda^{*}, \alpha_{o}^{*}\right)$ is a Pareto (strongly efficient) solution to problem (5).

Definition 5 (see [14]). Let $\left(\lambda^{*}, \alpha_{o}^{*}\right)$ be a feasible solution to problem (5). If there is no feasible solution $\left(\lambda, \alpha_{o}\right)$ of (5) such that $\alpha_{i o}<\alpha_{i o}^{*}$ for all $i=1, \ldots, m$, then it is said the $\left(\lambda^{*}, \alpha_{o}^{*}\right)$ is a weakly Pareto (efficient) solution to problem (5).

Theorem 6. Suppose that the DMU is ERM efficient and $\left(\lambda^{*}, \theta^{*}, \varphi^{*}\right)$ is an optimal solution to problem $(1)\left(\theta_{i}^{*}=1, \varphi_{r}^{*}=\right.$ 1 for all $i, r$ and $\left.\rho_{o}^{*}=1\right)$. Let $\left(\hat{\lambda}^{*}, \widehat{\alpha}_{o}^{*}\right)$ be a Pareto solution to problem (5) such that $\widehat{\alpha}_{o}^{*}>X_{o}$, and $\left(\lambda^{+*}, \theta^{+*}, \varphi^{+*}\right)$ is an optimal solution to problem (4) with the optimal value of $\rho_{o}^{+*}$. In addition, suppose that the optimal value and an optimal solution of the problem

$$
\begin{aligned}
& K_{o}^{*}=\max \frac{1}{s} \sum_{r=1}^{s} k_{r o} \\
& \text { s.t. } \quad \sum_{j=1}^{n}\left(\lambda_{j}^{+*}+\lambda_{n+1}^{+*} \widehat{\lambda}_{j}^{*}\right) y_{r j} \geq k_{r o} \varphi_{r}^{*} \beta_{r o}=k_{r o} \beta_{r o}, \\
& r=1, \ldots, s
\end{aligned}
$$

$$
k_{r o} \geq 1, \quad r=1, \ldots, s
$$

are $K_{o}^{*}$ and $k_{o}^{*}=\left(k_{1 o}^{*}, \ldots, k_{s o}^{*}\right)$, respectively. If the inputs of the $D M U_{o}$ are increased to $\widehat{\alpha}_{o}^{*}$, then (i) if $K_{o}^{*}=1$ (it is clear $k_{\text {ro }}^{*}=1$, for each $r$ ), then eff $\left(\widehat{\alpha}_{o}^{*}, \beta_{o}\right)=e f f\left(X_{o}, Y_{o}\right)$,

(ii) if $K_{o}^{*}>1$, then eff $\left(\widehat{\alpha}_{o}^{*}, k_{o}^{*} \star \beta_{o}\right)=\operatorname{eff}\left(X_{o}, Y_{o}\right)$.

Remark 7. $k_{o}^{*} \star \beta_{o}=\left(k_{1 o}^{*} \beta_{1 o}, \ldots, k_{s o}^{*} \beta_{s o}\right)$ and $\left(k_{o}^{*} \star \beta_{o}\right)-\beta_{o}=$ $\left(k_{1 o}^{*} \beta_{1 o}-\beta_{1 o}, \ldots, k_{s o}^{*} \beta_{s o}-\beta_{s o}\right)$. Note that the $k_{r o}^{*} \beta_{r o}-\beta_{r o}$ indicates the lack-output amount in $r$ th output component of the $\mathrm{DMU}_{o}$. In other words, for the decision maker to preserve the ERM-efficiency score of the $\mathrm{DMU}_{o}$ while the inputs increase from $X_{o}$ to $\widehat{\alpha}_{o}^{*}$, they are required to increase the outputs from $Y_{o}$ to $k_{o}^{*} \star \beta_{o}$.

Proof. Because $\left(\hat{\lambda}^{*}, \widehat{\alpha}_{o}^{*}\right)$ is a feasible solution of (5), the following inequalities are held:

$$
\begin{gathered}
\sum_{j=1}^{n} \widehat{\lambda}_{j}^{*} x_{i j} \leq \theta_{i}^{*} \widehat{\alpha}_{i o}^{*}=\widehat{\alpha}_{i o}^{*}, \quad i=1, \ldots, m \\
\sum_{j=1}^{n} \widehat{\lambda}_{j}^{*} y_{r j} \geq \varphi_{r}^{*} \beta_{r o}=\beta_{r o}, \quad r=1, \ldots, s \\
\widehat{\alpha}_{i o}^{*} \geq x_{i o}, \quad i=1, \ldots, m \\
\hat{\lambda}_{j}^{*} \geq 0, \quad j=1, \ldots, n .
\end{gathered}
$$

With regard to inequalities (7), (8), and (10) it is obvious that $\left(\bar{\lambda}, \theta^{*}, \varphi^{*}\right)$ is a feasible solution to problem $(4)$, where $\bar{\lambda}=$ $\left(\widehat{\lambda}^{*}, 0\right) \geq 0, \theta^{*}=\left(\theta_{1}^{*}, \ldots, \theta_{m}^{*}\right) \in R_{+}^{m}$, and $\varphi^{*}=\left(\varphi_{1}^{*}, \ldots, \varphi_{s}^{*}\right) \epsilon$ $R_{+}^{s}$, therefore, $\rho_{o}^{+*} \leq \rho_{o}^{*}$.

Using inequalities (7) and (8) in problem (4), the following results are obtained:

$$
\begin{gathered}
\theta_{i}^{+*} \widehat{\alpha}_{i o}^{*} \geq \sum_{j=1}^{n} \lambda_{j}^{+*} x_{i j}+\lambda_{n+1}^{+*} \widehat{\alpha}_{i o}^{*} \\
=\sum_{j=1}^{n} \lambda_{j}^{+*} x_{i j}+\lambda_{n+1}^{+*}\left(\sum_{j=1}^{n} \widehat{\lambda}_{j}^{*} x_{i j}\right), \\
\theta_{i}^{+*} \widehat{\alpha}_{i o}^{*} \geq \sum_{j=1}^{n}\left(\lambda_{j}^{+*}+\lambda_{n+1}^{+*} \widehat{\lambda}_{j}^{*}\right) x_{i j}, \quad i=1, \ldots, m \\
\varphi_{r}^{+*} \beta_{r o} \leq \sum_{j=1}^{n} \lambda_{j}^{+*} y_{r j}+\lambda_{n+1}^{+*} \beta_{r o} \\
\leq \sum_{j=1}^{n} \lambda_{j}^{+*} y_{r j}+\lambda_{n+1}^{+*}\left(\sum_{j=1}^{n} \hat{\lambda}_{j}^{*} y_{r j}\right) \\
\varphi_{r}^{+*} \beta_{r o} \leq \sum_{j=1}^{n}\left(\lambda_{j}^{+*}+\lambda_{n+1}^{+*} \widehat{\lambda}_{j}^{*}\right) y_{r j}, \quad r=1, \ldots, s .
\end{gathered}
$$

Set $\tilde{\lambda}_{j}=\lambda_{j}^{+*}+\lambda_{n+1}^{+*} \hat{\lambda}_{j}^{*}$ for each $j=1, \ldots, n$, and it is obviously seen that $\tilde{\lambda}=\left(\widetilde{\lambda}_{1}, \ldots, \tilde{\lambda}_{n}\right) \geq 0$.

Now by contradiction assume that $\rho_{o}^{+*}<\rho_{o}^{*}$. 
Since $\rho_{o}^{+*}<\rho_{o}^{*}$, then $(1 / m) \sum_{i=1}^{m} \theta_{i}^{+*}<1$ or $(1 / s) \sum_{r=1}^{s} \varphi_{r}^{+*}>$ 1 , so that there are two cases.

(a) Let $(1 / m) \sum_{i=1}^{m} \theta_{i}^{+*}<1$. There exists at least one $t, 1 \leq$ $t \leq m$, such that $\theta_{t}^{+*}<1$. With regard to inequalities (11), the following inequality is obtained:

$$
\sum_{j=1}^{n} \tilde{\lambda}_{j} x_{t j} \leq \theta_{t}^{+*} \widehat{\alpha}_{t o}^{*}<\widehat{\alpha}_{t o}^{*}
$$

On the other hand, since, for each $i=1, \ldots, m, \widehat{\alpha}_{i o}^{*}>x_{i o}$, therefore if

$$
\eta_{t}=\min \left\{\widehat{\alpha}_{t o}^{*}-\sum_{j=1}^{n} \tilde{\lambda}_{j} x_{t j}, \widehat{\alpha}_{t o}^{*}-x_{t o}\right\},
$$

then $\eta_{t}>0$. Now define

$$
\widetilde{\alpha}_{i o}= \begin{cases}\widehat{\alpha}_{i o}^{*}, & \text { if } i \neq t, \\ \widehat{\alpha}_{i o}^{*}-\eta_{t}, & \text { if } i=t .\end{cases}
$$

Taking (14) into consideration, the following inequalities are obtained:

$$
\begin{gathered}
\eta_{t} \leq \widehat{\alpha}_{t o}^{*}-\sum_{j=1}^{n} \widetilde{\lambda}_{j} x_{t j} \Longrightarrow \sum_{j=1}^{n} \widetilde{\lambda}_{j} x_{t j} \leq \widehat{\alpha}_{t o}^{*}-\eta_{t}=\theta_{t}^{*} \widetilde{\alpha}_{t o}, \\
\eta_{t} \leq \widehat{\alpha}_{t o}^{*}-x_{t o} \Longrightarrow x_{t o} \leq \widehat{\alpha}_{t o}^{*}-\eta_{t}, \Longrightarrow x_{t o} \leq \widetilde{\alpha}_{t o}
\end{gathered}
$$

which implies that $\tilde{\alpha}_{o} \geq X_{o}$.

Based on $\tilde{\lambda} \geq 0$ and (11), (12), and (16) and $\widetilde{\alpha}_{o} \geq X_{o},\left(\tilde{\lambda}, \widetilde{\alpha}_{o}\right)$ is a feasible solution to problem (5), where $\widetilde{\alpha}_{i o} \leq \widehat{\alpha}_{i o}^{*}$ for all $i=$ $1, \ldots, m$, and $\widetilde{\alpha}_{i o}<\widehat{\alpha}_{i o}^{*}$ for some $i=1, \ldots, m$. This contradicts the assumption that $\left(\widehat{\lambda}^{*}, \widehat{\alpha}_{o}^{*}\right)$ is a Pareto solution to problem (5).

(b) $\operatorname{Let}(1 / s) \sum_{r=1}^{s} \varphi_{r}^{+*}>1$. There exists at least one $t, 1 \leq t \leq s$, such that $\varphi_{t}^{+*}>1$. By (12), the following strict inequality is obtained:

$$
\sum_{j=1}^{n} \widetilde{\lambda}_{j} y_{t j} \geq \varphi_{t}^{+*} \beta_{t o}>\beta_{t o}
$$

and, therefore, there exists a $\mu_{t}>1$ that satisfies

$$
\sum_{j=1}^{n} \widetilde{\lambda}_{j} y_{t j} \geq \mu_{t} \beta_{t o}=\left(\mu_{t} k_{t o}^{*}\right) \beta_{t o} .
$$

Now define

$$
\tilde{k}_{r o}= \begin{cases}k_{r o}^{*}=1, & \text { if } r \neq t, \\ k_{r o}^{*} \mu_{t}=\mu_{t}, & \text { if } r=t .\end{cases}
$$

It is obvious that $\widetilde{k}_{o}=\left(\widetilde{k}_{10}, \ldots, \widetilde{k}_{s o}\right)$ is a feasible solution to problem (6), such that

$$
K_{o}^{*}=\frac{1}{s} \sum_{r=1}^{s} k_{r o}<\frac{1}{s} \sum_{r=1}^{s} \widetilde{k}_{r o}=\widetilde{K}_{o} .
$$

This contradicts the assumption expressing that the optimal value of problem (6) is $K_{o}^{*}$. Consequently, in each case $\rho_{o}^{+*} \nless \rho_{o}^{*}$, and since $\rho_{o}^{+*} \leqslant \rho_{o}^{*}$, therefore, $\rho_{o}^{+*}=\rho_{o}^{*}$.

(ii) with replacing the notation $\beta_{\text {ro }}$ by $k_{\text {ro }}^{*} \beta_{\text {ro }}$ for each $r=$ $1, \ldots, s$, in problem (6), clearly, optimal value of problem (6) is 1 . Therefore, according to part (i), then $\operatorname{eff}\left(\widehat{\alpha}_{o}^{*}, k_{o}^{*} \star \beta_{o}\right)=$ $\operatorname{eff}\left(X_{o}, Y_{o}\right)$.

Although Theorem 6 satisfies for all units that be ERMefficient, but the following theorem is the converse version of Theorem 6 that satisfies for all $\mathrm{DMU}_{s}$.

Theorem 8. Suppose that the $\left(\lambda^{*}, \theta^{*}, \varphi^{*}\right)$ is an optimal solution to problem (1) with the optimal value of $\rho_{o}^{*}$. Let $\left(\bar{\lambda}, \bar{\alpha}_{o}\right)$ be a feasible solution to problem (5). If eff $\left(\bar{\alpha}_{o}, \beta_{o}\right)=\operatorname{eff}\left(X_{o}, Y_{o}\right)$, then $\left(\bar{\lambda}, \bar{\alpha}_{o}\right)$ must be a Pareto solution to problem (5).

Proof. If $\left(\bar{\lambda}, \bar{\alpha}_{o}\right)$ is not a Pareto solution to problem (5), then there would exist another feasible solution of (5), $\left(\widetilde{\lambda}, \widetilde{\alpha}_{o}\right)$ in which, $\widetilde{\alpha}_{i o} \leq \bar{\alpha}_{i o}$ for $i=1, \ldots, m$ and $\widetilde{\alpha}_{i o}<\bar{\alpha}_{i o}$ for at least one $i \in\{1, \ldots, m\}$. Let $I=\left\{i \mid \widetilde{\alpha}_{i o}<\bar{\alpha}_{i o}\right\}$. Then

$$
\begin{gathered}
\sum_{j=1}^{n} \widetilde{\lambda}_{j} x_{i j} \leq \theta_{i}^{*} \widetilde{\alpha}_{i o}<\theta_{i}^{*} \bar{\alpha}_{i o}, \quad i \in I, \\
\sum_{j=1}^{n} \widetilde{\lambda}_{j} x_{i j} \leq \theta_{i}^{*} \widetilde{\alpha}_{i o} \leq \theta_{i}^{*} \bar{\alpha}_{i o}, \quad i \notin I, \\
\sum_{j=1}^{n} \widetilde{\lambda}_{j} y_{r j} \geq \varphi_{r}^{*} \beta_{r o}, \quad r=1, \ldots, s, \\
\tilde{\lambda}_{j} \geq 0, \quad j=1, \ldots, n .
\end{gathered}
$$
that

By inequality (22) there exists $\mu_{i}<1$ for all $i \in I$, such

$$
\sum_{j=1}^{n} \widetilde{\lambda}_{j} x_{i j} \leq\left(\mu_{i} \theta_{i}^{*}\right) \bar{\alpha}_{i o}, \quad i \in I .
$$

For each $i=1, \ldots, m$ and $r=1, \ldots, s$, define

$$
\bar{\theta}_{i}=\left\{\begin{array}{ll}
\mu_{i} \theta_{i}^{*} & \text { if } i \in I, \\
\theta_{i}^{*}, & \text { if } i \notin I,
\end{array} \quad \bar{\varphi}_{r}=\varphi_{r}^{*}, \quad r=1, \ldots, s .\right.
$$

Based on inequalities (23)-(26), $(\tilde{\lambda}, \bar{\theta}, \bar{\varphi})$ is a feasible solution to problem (1) (only restrictions on the right have replaced the $X_{o}$ and $Y_{o}$ by $\bar{\alpha}_{o}$ and $\beta_{o}$ in (1), resp.), where $\bar{\theta}=\left(\bar{\theta}_{1}, \ldots, \bar{\theta}_{m}\right)$ and $\bar{\varphi}=\left(\bar{\varphi}_{1}, \ldots, \bar{\varphi}_{s}\right)$. Therefore

$$
\bar{\rho}_{o}=\frac{(1 / m) \sum_{i=1}^{m} \bar{\theta}_{i}}{(1 / s) \sum_{r=1}^{s} \bar{\varphi}_{r}}<\frac{(1 / m) \sum_{i=1}^{m} \theta_{i}^{*}}{(1 / s) \sum_{r=1}^{s} \varphi_{r}^{*}}=\rho_{o}^{*},
$$

which is against the assumption expressing that $\operatorname{eff}\left(\bar{\alpha}_{o}, \beta\right)=$ $\rho_{o}^{*}$.

Along with Theorem 2 in Section 5 in [14] the following theorem is considered. 
Theorem 9. Suppose that the $D M U_{o}$ is ERM efficient and the inputs and the outputs for $D M U_{o}$ are going to increase from $X_{o}$ and $Y_{o}$ to $\alpha_{o}=X_{o}+\Delta X_{o}$ and $\beta_{o}=Y_{o}+\Delta Y_{o}$, respectively, where there are $\Delta X_{o} \supsetneqq 0, \Delta Y_{o} \gtreqless 0$. It is worth mentioning that it is required that the $\left(\alpha_{o}, \beta_{o}\right)$ belongs to the production possibility set. Consider the following problem:

$$
\begin{aligned}
\hat{\rho}_{o}=\min & \frac{(1 / m) \sum_{i=1}^{m} \theta_{i}}{(1 / s) \sum_{r=1}^{s} \varphi_{r}} \\
\text { s.t. } \quad & \sum_{j=1}^{n} \lambda_{j} x_{i j} \leq \theta_{i} \alpha_{i o}, \quad i=1, \ldots, m \\
& \sum_{j=1}^{n} \lambda_{j} y_{r j} \geq \varphi_{r} \beta_{r o}, \quad r=1, \ldots, s \\
& \theta_{i} \leq 1, \quad \varphi_{r} \geq 1, \quad i=1, \ldots, m, \quad r=1, \ldots, s \\
& \lambda_{j} \geq 0, \quad j=1, \ldots, n .
\end{aligned}
$$

If the inputs and outputs of DMU $U_{o}$ are increased from $X_{o}$ to $\widehat{X}_{o}=\left(\widehat{\theta}_{1} x_{1 o}, \ldots, \widehat{\theta}_{m} x_{m o}\right)$ and $\widehat{Y}_{o}=\left(\widehat{\varphi}_{1} y_{1 o}, \ldots, \widehat{\varphi}_{s} y_{s o}\right)$, respectively, and $(\hat{\lambda}, \hat{\theta}, \widehat{\varphi})$ is an optimal solution to problem (29) with the optimal value of $\hat{\rho}$, then eff $\left(X_{o}, Y_{o}\right)=\operatorname{eff}\left(\widehat{X}_{o}, \widehat{Y}_{o}\right)$.

Proof. When the inputs and outputs of the $\mathrm{DMU}_{o}$ convert to $\widehat{X}_{o}$ and $\widehat{Y}_{o}$, respectively, the ERM-efficiency score of the DMU equals the optimal value of the problem below

$$
\begin{aligned}
\tilde{\rho}_{o}=\min & \frac{(1 / m) \sum_{i=1}^{m} \theta_{i}}{(1 / s) \sum_{r=1}^{s} \varphi_{r}} \\
\text { s.t. } \quad & \sum_{j=1}^{n} \lambda_{j} x_{i j}+\lambda_{n+1} \widehat{x}_{i o} \leq \theta_{i} \widehat{x}_{i o}, \quad i=1, \ldots, m \\
& \sum_{j=1}^{n} \lambda_{j} y_{r j}+\lambda_{n+1} \widehat{y}_{r o} \geq \varphi_{r} \widehat{y}_{r o}, \quad r=1, \ldots, s \\
& \theta_{i} \leq 1, \quad \varphi_{r} \geq 1, \quad i=1, \ldots, m, \quad r=1, \ldots, s \\
& \lambda_{j} \geq 0, \quad j=1, \ldots, n+1 .
\end{aligned}
$$

Suppose that $(\tilde{\lambda}, \widetilde{\theta}, \widetilde{\varphi})$ is an optimal solution to problem (30) with the optimal value of $\widetilde{\rho}_{o}$. To prove the theorem, $\widetilde{\rho}_{o}=1$ should be shown. By contradiction assume that $\tilde{\rho}_{o}<1$.

Problem (30) is nonlinear, but it can be converted to the following linear programming problem by employing the $t=$ $1 /(1 / s) \sum_{r=1}^{s} \varphi_{r}>0$ :

$$
\begin{aligned}
\overline{\tilde{\rho}}_{o}=\min & \frac{1}{m} \sum_{i=1}^{m} \bar{\theta}_{i} \\
\text { s.t. } \quad & \sum_{j=1}^{n} \bar{\lambda}_{j} x_{i j}+\bar{\lambda}_{n+1} \widehat{x}_{i o} \leq \bar{\theta}_{i} \widehat{x}_{i o}, \quad i=1, \ldots, m \\
& \sum_{j=1}^{n} \bar{\lambda}_{j} y_{r j}+\bar{\lambda}_{n+1} \widehat{y}_{r o} \geq \bar{\varphi}_{r} \widehat{y}_{r o}, \quad r=1, \ldots, s
\end{aligned}
$$

$$
\begin{aligned}
& \sum_{r=1}^{s} \bar{\varphi}_{r}=s, \quad t \geq \epsilon, \\
& \bar{\theta}_{i} \leq t, \quad \bar{\varphi}_{r} \geq t, \quad i=1, \ldots, m, \quad r=1, \ldots, s \\
& \bar{\lambda}_{j} \geq 0, \quad j=1, \ldots, n+1,
\end{aligned}
$$

where $(\bar{\theta}, \bar{\varphi})=(t \theta, t \varphi), \bar{\lambda}_{j}=t \lambda_{j}$ for $j=1, \ldots, n$, and $\epsilon$ is nonArchimedean infinitesimal. Note that $t \geq \epsilon$ is a redundant constraint in problem (31) and hence can be omitted. The dual problem (31) is as follows:

$$
\begin{array}{ll}
\max & s z \\
\text { s.t. } & U^{t} Y_{j}-V^{t} X_{j} \leq 0, \quad j=1, \ldots, n \\
& U^{t} \widehat{Y}_{o}-V^{t} \widehat{X}_{o} \leq 0, \\
& v_{i} \widehat{x}_{i o}-\tau_{i}=\frac{1}{m}, \quad i=1, \ldots, m \\
& -u_{r} \widehat{y}_{r o}+w_{r}+z=0, \quad r=1, \ldots, s \\
& e_{m} \tau-e_{s} W=0, \\
& V \geq 0, \quad U \geq 0, \quad \tau \geq 0, \quad z \in R,
\end{array}
$$

where $e_{m}=(1, \ldots, 1) \in R^{m}$ and $e_{s}=(1, \ldots, 1) \in R^{s}$. Assume $(\bar{V}, \bar{U}, \bar{\tau}, \bar{W}, \bar{z})$ is an optimal solution to the above problem. Based on the duality Theorem, the following relation is held:

$$
s \bar{z}=\widetilde{\rho}_{o}<1 .
$$

Also, constraint sets (33) are added together and result in

$$
-\bar{U}^{t} \widehat{Y}_{o}+e_{s} \bar{W}+s \bar{z}+\bar{V}^{t} \widehat{X}_{o}-e_{m} \bar{\tau}=1
$$

taking (35) into consideration, the following inequality is obtained:

$$
s \bar{z}=\tilde{\rho}_{o}<1=-\bar{U}^{t} \widehat{Y}_{o}+e_{s} \bar{W}+s \bar{z}+\bar{V}^{t} \widehat{X}_{o}-e_{m} \bar{\tau}
$$

and, therefore

$$
\bar{U}^{t} \widehat{Y}_{o}-\bar{V}^{t} X_{o}<0
$$

Since the variable $\bar{\lambda}_{n+1}$ is corresponding to the constraint (32) in the above problem that is unbinding at each optimal solution, therefore, according to the complementary slackness theorem, $\bar{\lambda}_{n+1}$ must be 0 , and so $\lambda_{n+1}=0$. 
Consequently, $\tilde{\rho}_{o}$ is also the optimal value of the following problem:

$$
\begin{aligned}
\tilde{\rho}_{o}=\min & \frac{(1 / m) \sum_{i=1}^{m} \theta_{i}}{(1 / s) \sum_{r=1}^{s} \varphi_{r}} \\
\text { s.t. } \quad & \sum_{j=1}^{n} \lambda_{j} x_{i j} \leq \theta_{i} \widehat{x}_{i o}, \quad i=1, \ldots, m \\
& \sum_{j=1}^{n} \lambda_{j} y_{r j} \geq \varphi_{r} \widehat{y}_{r o}, \quad r=1, \ldots, s \\
& \theta_{i} \leq 1, \quad \varphi_{r} \geq 1, \quad i=1, \ldots, m, \quad r=1, \ldots, s \\
& \lambda_{j} \geq 0, \quad j=1, \ldots, n .
\end{aligned}
$$

Now, consider the following problem:

$$
\begin{aligned}
\bar{\rho}_{o}=\min & \frac{(1 / m) \sum_{i=1}^{m} \widehat{\theta}_{i} \theta_{i}}{(1 / s) \sum_{r=1}^{s} \widehat{\varphi}_{r} \varphi_{r}} \\
\text { s.t. } \quad & \sum_{j=1}^{n} \lambda_{j} x_{i j} \leq \widehat{\theta}_{i} \theta_{i} x_{i o}, \quad i=1, \ldots, m \\
& \sum_{j=1}^{n} \lambda_{j} y_{r j} \geq \widehat{\varphi}_{r} \varphi_{r} y_{r o}, \quad r=1, \ldots, s \\
& \widehat{\theta}_{i} \theta_{i} \leq 1, \quad \widehat{\varphi}_{r} \varphi_{r} \geq 1, \quad i=1, \ldots, m, \quad r=1, \ldots, s \\
& \lambda_{j} \geq 0, \quad j=1, \ldots, n .
\end{aligned}
$$

Clearly, $(\tilde{\lambda}, \widetilde{\theta}, \widetilde{\varphi})$ is a feasible solution to problem (40), and so

$$
\bar{\rho}_{o} \leq \frac{(1 / m) \sum_{i=1}^{m} \widehat{\theta}_{i} \tilde{\theta}_{i}}{(1 / s) \sum_{r=1}^{s} \widehat{\varphi}_{r} \widetilde{\varphi}_{r}}
$$

On the other hand, since $\tilde{\rho}_{o}<1$, there exists at least one $t$, $1 \leq t \leq m$ or $1 \leq t \leq s$, such that $\widetilde{\theta}_{t}<1$, or $\widetilde{\varphi}_{t}>1$. Therefore, in each case

$$
\bar{\rho}_{o} \leq \frac{(1 / m) \sum_{i=1}^{m} \widehat{\theta}_{i} \widetilde{\theta}_{i}}{(1 / s) \sum_{r=1}^{s} \widehat{\varphi}_{r} \widetilde{\varphi}_{r}}<\frac{(1 / m) \sum_{i=1}^{m} \widehat{\theta}_{i}}{(1 / s) \sum_{r=1}^{s} \widehat{\varphi}_{r}}=\widehat{\rho}_{o} .
$$

Obviously, problem (40) is just problem (29) (replacing the notation $\widehat{\theta}_{i} \theta_{i}$ by $\theta_{i}$ and $\widehat{\varphi}_{r} \varphi_{r}$ by $\left.\varphi_{r}\right)$. Therefore, in each case, the optimal value of problem (29) would be $\bar{\rho}_{o}<\hat{\rho}_{o}$, which contradicts the fact that the maximum value of (29) is $\hat{\rho}_{o}$.

\section{Estimate Outputs}

In this section, the problem provided by Wei et al. [14] is considered. Based on the results of their study, this question is addressed: suppose that the $\mathrm{DMU}_{o}$ is ERM efficient, if the ERM-efficiency score of $\mathrm{DMU}_{o}$ remains unchanged while the inputs increase, and how much should the outputs of the $\mathrm{DMU}_{o}$ increase?

To solve the above problem, to the end of this section, presume that the inputs of $\mathrm{DMU}_{o}$ are increased from $X_{o}$ to $\alpha_{o}=X_{o}+\Delta X_{o}$, where $\Delta X_{o} \supsetneqq 0$. The aim of the problem is to estimate the output vector provided that the $\mathrm{DMU}_{o}$ is still ERM efficient. In fact,

$$
\beta_{o}^{*}=\left(\beta_{1 o}^{*}, \beta_{2 o}^{*}, \ldots, \beta_{s o}^{*}\right)^{t}=Y_{o}+\Delta Y_{o}, \quad \Delta Y_{o} \supsetneqq 0 .
$$

Assume $\mathrm{DMU}_{n+1}$ represents $\mathrm{DMU}_{o}$ after modification of the inputs and outputs. Along the line of [14], the following model is considered to calculate the ERM efficiency of $\mathrm{DMU}_{n+1}$ :

$$
\begin{aligned}
\rho_{o}^{+*}=\min \quad & \frac{(1 / m) \sum_{i=1}^{m} \theta_{i}}{(1 / s) \sum_{r=1}^{s} \varphi_{r}} \\
\text { s.t. } \quad & \sum_{j=1}^{n} \lambda_{j} x_{i j}+\alpha_{i o} \lambda_{n+1} \leq \theta_{i} \alpha_{i o}, \quad i=1, \ldots, m \\
& \sum_{j=1}^{n} \lambda_{j} y_{r j}+\beta_{r o}^{*} \lambda_{n+1} \geq \varphi_{r} \beta_{r o}^{*}, \quad r=1, \ldots, s \\
& \theta_{i} \leq 1, \quad \varphi_{r} \geq 1, \quad i=1, \ldots, m, \quad r=1, \ldots, s \\
& \lambda_{j} \geq 0, \quad j=1, \ldots, n+1 .
\end{aligned}
$$

Based on Definition 3 it is said that the ERM efficiency remains unchanged, if and only if $\operatorname{eff}\left(\alpha_{o}, \beta_{o}^{*}\right)=\operatorname{eff}\left(X_{o}, Y_{o}\right)$. To answer the above question, along the line of [14], the following MOLP model is considered:

$$
\begin{array}{ll}
\max & \left(\beta_{1 o}, \ldots, \beta_{s o}\right) \\
\text { s.t. } & \sum_{j=1}^{n} \lambda_{j} x_{i j} \leq \theta_{i}^{*} \alpha_{i o}, \quad i=1, \ldots, m \\
& \sum_{j=1}^{n} \lambda_{j} y_{r j} \geq \varphi_{r}^{*} \beta_{r o}, \quad r=1, \ldots, s \\
& \alpha_{i o} \geq x_{i o}, \quad i=1, \ldots, m \\
& \lambda_{j} \geq 0, \quad j=1, \ldots, n,
\end{array}
$$

where $\left(\theta^{*}, \varphi^{*}\right)$ is an optimal solution to problem (1).

Theorem 10. Suppose that the $D M U_{o}$ is ERM efficient and $\left(\lambda^{*}, \theta^{*}, \varphi^{*}\right)$ is an optimal solution to problem $(1)$. Let $\left(\widehat{\lambda}^{*}, \widehat{\beta}_{o}^{*}\right)$ be a Pareto solution to problem (45) such that $\widehat{\beta}_{o}^{*}>Y_{o}$, and $\left(\lambda^{+*}, \theta^{+*}, \varphi^{+*}\right)$ is an optimal solution to problem (4) with 
the optimal value of $\rho_{o}^{+*}$. Also, suppose that the optimal value and an optimal solution of the following problem:

$$
\begin{aligned}
K_{o}^{*}=\max & \frac{1}{m} \sum_{i=1}^{m} k_{i o} \\
\text { s.t. } \quad & \sum_{j=1}^{n}\left(\lambda_{j}^{+*}+\lambda_{n+1}^{+*} \hat{\lambda}_{j}^{*}\right) x_{i j} \leq k_{i o} \theta_{i}^{*} \alpha_{i o}=k_{i o} \alpha_{i o}, \\
& k_{i o} \leq 1, \quad i=1, \ldots, m
\end{aligned}
$$

are $K_{o}^{*}$ and $k_{o}^{*}=\left(k_{1 o}^{*}, \ldots, k_{m o}^{*}\right)$. If the outputs of $D M U_{o}$ are increased to $\widehat{\beta}_{o}^{*}$, then

(i) if $K_{o}^{*}=1$, then eff $\left(\alpha_{o}, \widehat{\beta}_{o}^{*}\right)=\operatorname{eff}\left(X_{o}, Y_{o}\right)$,

(ii) if $K_{o}^{*}<1$, then eff $\left(k_{o}^{*} \star \alpha_{o}, \widehat{\beta}_{o}^{*}\right)=\operatorname{eff}\left(X_{o}, Y_{o}\right)$.

Remark 11. $k_{o}^{*} \star \alpha_{o}=\left(k_{1 o}^{*} \alpha_{1 o}, \ldots, k_{m o}^{*} \alpha_{m o}\right)$ and $\alpha_{o}-\left(k_{o}^{*} \star \alpha_{o}\right)=$ $\left(\alpha_{1 o}-k_{1 o}^{*} \alpha_{1 o}, \ldots, \alpha_{m o}-k_{m o}^{*} \alpha_{m o}\right)$. Note that the $\alpha_{i o}-k_{i o}^{*} \alpha_{i o}$ indicates extra-input amount in $i$ th input component of the DMU. In other words, for the decision maker to preserve the ERM-efficiency score of the $\mathrm{DMU}_{o}$ while the outputs increase from $Y_{o}$ to $\widehat{\beta}_{o}^{*}$, they are required to increase the inputs from $X_{o}$ to $k_{o}^{*} \star \alpha_{o}$.

Proof. The proof is similar to the proof of Theorem 6.

Theorem 12. Assume $\left(\lambda^{*}, \theta^{*}, \varphi^{*}\right)$ is an optimal solution to problem (1) with the optimal value of $\rho_{o}^{*}$. Let $\left(\bar{\lambda}, \bar{\beta}_{o}\right)$ be a feasible solution to problem (45). If eff $\left(\alpha_{o}, \bar{\beta}_{o}\right)=\operatorname{eff}\left(X_{o}, Y_{o}\right)$, then $\left(\bar{\lambda}, \bar{\beta}_{o}\right)$ must be a Pareto solution to problem (45).

Proof. The proof is similar to the proof of Theorem 8 .

\section{Estimate the Minimum Increase of Inputs and the Maximum Increase of Outputs}

In order to present suitable patterns to the decision maker to increase inputs and outputs for an ERM-efficient DMU, under preserving the ERM-efficiency index, this new question in field of inverse DEA is addressed: if $\mathrm{DMU}_{o}$ is ERM efficient, while the inputs and outputs are required to be increased, how much should the inputs and outputs of the $\mathrm{DMU}_{o}$ be increased? In other words, under preserving the ERM-efficiency score, how much should the minimum and maximum of input and output vectors of the $\mathrm{DMU}_{o}$ increase, respectively?

By answering the question, the decision maker may be able to take better decisions in order to extend decision making units. That is to say that the decision maker can take necessary actions by choosing a suitable strategy for spreading an ERM-efficient DMU.
The aim of addressing this question is estimating the minimum increase of inputs and the maximum increase of outputs provided that the $\mathrm{DMU}_{o}$ is still ERM efficient. In fact,

$$
\begin{gathered}
\alpha_{o}^{*}=\left(\alpha_{1 o}^{*}, \alpha_{2 o}^{*}, \ldots, \alpha_{m o}^{*}\right)^{t}=X_{o}+\Delta X_{o}, \quad \Delta X_{o} \supsetneqq 0, \\
\beta_{o}^{*}=\left(\beta_{1 o}^{*}, \beta_{2 o}^{*}, \ldots, \beta_{s o}^{*}\right)^{t}=Y_{o}+\Delta Y_{o}, \quad \Delta Y_{o} \supsetneqq 0 .
\end{gathered}
$$

Remark 13. Note that it is required to make the rate of increase inputs and outputs of the unit under assessment bounded; otherwise, it is possible that at least one of the components of $\alpha_{o}^{*}$ or $\beta_{o}^{*}$ is unbounded.

Suppose that $\mathrm{DMU}_{n+1}$ represents $\mathrm{DMU}_{o}$ after modification of the inputs and outputs. Based on results of $[14,16]$, the following model is considered to estimate the ERM efficiency of $\mathrm{DMU}_{n+1}$ :

$$
\begin{aligned}
\rho_{o}^{+*}=\min \quad & \frac{(1 / m) \sum_{i=1}^{m} \theta_{i}}{(1 / s) \sum_{r=1}^{s} \varphi_{r}} \\
\text { s.t. } \quad & \sum_{j=1}^{n} \lambda_{j} x_{i j}+\alpha_{i o}^{*} \lambda_{n+1} \leq \theta_{i} \alpha_{i o}^{*}, \quad i=1, \ldots, m \\
& \sum_{j=1}^{n} \lambda_{j} y_{r j}+\beta_{r o}^{*} \lambda_{n+1} \geq \varphi_{r} \beta_{r o}^{*}, \quad r=1, \ldots, s \\
& \theta_{i} \leq 1, \quad \varphi_{r} \geq 1, \quad i=1, \ldots, m, \quad r=1, \ldots, s \\
& \lambda_{j} \geq 0, \quad j=1, \ldots, n+1 .
\end{aligned}
$$

Definition 14. If the optimal values of problems (1) and (48) are equal, it is said that the ERM efficiency remains unchanged; that is, $\operatorname{eff}\left(\alpha_{o}^{*}, \beta_{o}^{*}\right)=\operatorname{eff}\left(X_{o}, Y_{o}\right)$.

To solve this new question, that is, to estimate the minimum increase of inputs and the maximum increase of outputs, along the line of $[14,16]$, the following MOLP problem is considered:

$$
\begin{array}{ll}
\min & \left(\alpha_{1 o}, \ldots, \alpha_{m o}\right) \\
\max & \left(\beta_{1 o}, \ldots, \beta_{s o}\right) \\
\text { s.t. } & \sum_{j=1}^{n} \lambda_{j} x_{i j} \leq \theta_{i}^{*} \alpha_{i o}, \quad i=1, \ldots, m \\
& \sum_{j=1}^{n} \lambda_{j} y_{r j} \geq \varphi_{r}^{*} \beta_{r o}, \quad r=1, \ldots, s \\
& \alpha_{i o} \geq x_{i o}, \quad i=1, \ldots, m \\
& \beta_{r o} \geq y_{r o}, \quad r=1, \ldots, s \\
& \beta_{o} \in \Gamma, \quad \alpha_{o} \in \Lambda, \\
& \lambda_{j} \geq 0, \quad j=1, \ldots, n,
\end{array}
$$

where $\theta^{*}$ and $\varphi^{*}$ are an optimal solution to problem (1), $\Gamma$ and $\Lambda$ are bounded sets, and show the maximum rate of increase 
in inputs and outputs of the $\mathrm{DMU}_{o}$, respectively, such that by the decision maker are considered.

Theorem 15. Suppose that the $D M U_{o}$ is ERM efficient and $\left(\lambda^{*}, \theta^{*}, \varphi^{*}\right)$ is an optimal solution to problem (1). Let $\left(\widehat{\lambda}^{*}, \widehat{\alpha}_{o}^{*}, \widehat{\beta}_{o}^{*}\right)$ be a Pareto solution to problem (49) such that $\widehat{\alpha}_{o}^{*}>$ $X_{o}, \widehat{\beta}_{o}^{*} \supsetneqq Y_{o}$. If the inputs and outputs of $D M U_{o}$ are increased to $\widehat{\alpha}_{o}^{*}$ and $\widehat{\beta}_{o}^{*}$, respectively, then eff $\left(\widehat{\alpha}_{o}^{*}, \widehat{\beta}_{o}^{*}\right)=\operatorname{eff}\left(X_{o}, Y_{o}\right)$.

Proof. Assume $\left(\lambda^{+*}, \theta^{+*}, \varphi^{+*}\right)$ is an optimal solution to problem (48) with the optimal value of $\rho^{+*}$. The proof is similar to the proof of Theorem 6 , when replacing the notation $\beta_{o}$ by $\widehat{\beta}_{o}^{*}$. The only difference is in case (b), that is, when $(1 / s) \sum_{r=1}^{s} \varphi_{r}^{+*}>1$. In this case, there exists at least one $p$, $1 \leq p \leq s$, such that $\varphi_{p}^{+*}>1$. Taking (12) into consideration, the following inequality is obtained:

$$
\sum_{j=1}^{n} \widetilde{\lambda}_{j} y_{p j} \geq \varphi_{p}^{+*} \widehat{\beta}_{p o}^{*}>\widehat{\beta}_{p o}^{*}
$$

and, thus, there exists a $\eta_{p}>0$ that satisfies

$$
\sum_{j=1}^{n} \tilde{\lambda}_{j} y_{p j} \geq\left(\widehat{\beta}_{p o}^{*}+\eta_{p}\right)
$$

Now, define

$$
\tilde{\beta}_{r o}= \begin{cases}\beta_{r o}^{*}, & \text { if } r \neq p, \\ \eta_{r}+\widehat{\beta}_{r o}^{*}, & \text { if } r=p .\end{cases}
$$

Based on $\tilde{\lambda} \geq 0$, inequality (9), (11), and (51), and $\widetilde{\beta}_{o} \supsetneqq$ $\widehat{\beta}_{o}^{*} \supsetneqq Y_{o},\left(\tilde{\lambda}, \widehat{\alpha}_{o}^{*}, \widetilde{\beta}_{o}\right)$ is a feasible solution to problem (49), where $\widetilde{\beta}_{r o} \geq \widehat{\beta}_{r o}^{*}$ for all $r=1, \ldots, s$, and $\widetilde{\beta}_{r o}>\beta_{r o}^{*}$ for some $r=$ $1, \ldots, s$. This contradicts the assumption expressing that the $\left(\widehat{\lambda}^{*}, \widehat{\alpha}_{o}^{*}, \widehat{\beta}_{o}^{*}\right)$ is a Pareto solution to problem (49). Therefore, in any two cases, because $\rho_{o}^{+*} \nless \rho_{o}^{*}$ and $\rho_{o}^{+*} \leqslant \rho_{o}^{*}$, therefore $\rho_{o}^{+*}=\rho_{o}^{*}$.

Theorem 16. Suppose that the $\left(\lambda^{*}, \theta^{*}, \varphi^{*}\right)$ is an optimal solution to problem (1) with the optimal value of $\rho_{o}^{*}$. Let $\left(\bar{\lambda}, \bar{\alpha}_{o}, \bar{\beta}_{o}\right)$ be a feasible solution to the problem (49). If eff $\left(\bar{\alpha}_{o}, \bar{\beta}_{o}\right)=$ eff $\left(X_{o}, Y_{o}\right)$, then $\left(\bar{\lambda}, \bar{\alpha}_{o}, \bar{\beta}_{o}\right)$ must be a Pareto solution to problem (49).

Proof. If $\left(\bar{\lambda}, \bar{\alpha}_{o}, \bar{\beta}_{o}\right)$ is not a Pareto solution to problem (49), then there would exist another feasible solution of problem (5), $\left(\tilde{\lambda}, \tilde{\alpha}_{o}, \widetilde{\beta}\right)$, such that $\widetilde{\alpha}_{i o} \leq \bar{\alpha}_{i o}$ for all $i$ and $\widetilde{\beta}_{r o} \geq \bar{\beta}_{r o}$ for all $r$; furthermore, there exists at least one $i$ in which $\widetilde{\alpha}_{i o}<\bar{\alpha}_{i o}$ or at least one $r$ such that $\tilde{\beta}_{r o}>\bar{\beta}_{r o}$. Let $I=\left\{i \mid \widetilde{\alpha}_{i o}<\bar{\alpha}_{i o}\right\}$ and $O=\left\{r \mid \widetilde{\beta}_{r o}>\bar{\beta}_{r o}\right\}$. Therefore

$$
\begin{aligned}
& \sum_{j=1}^{n} \widetilde{\lambda}_{j} x_{i j} \leq \theta_{i}^{*} \widetilde{\alpha}_{i o}<\theta_{i}^{*} \bar{\alpha}_{i o} \quad i \in I \\
& \sum_{j=1}^{n} \widetilde{\lambda}_{j} x_{i j} \leq \theta_{i}^{*} \widetilde{\alpha}_{i o} \leq \theta_{i}^{*} \bar{\alpha}_{i o} \quad i \notin I
\end{aligned}
$$

TABLE 1: The data and ERM efficiency in Example 1.

\begin{tabular}{lccccccccc}
\hline DMUs & $x_{1}$ & $x_{2}$ & $y_{1}$ & $y_{2}$ & $\theta_{1}^{*}$ & $\theta_{2}^{*}$ & $\varphi_{1}^{*}$ & $\varphi_{2}^{*}$ & $\rho^{*}$ \\
\hline$A$ & 4.00 & 2.00 & 4.00 & 4.00 & 1.00 & 1.00 & 4.00 & 2.00 & 0.33 \\
$B$ & 2.00 & 1.00 & 8.00 & 4.00 & 1.00 & 1.00 & 1.00 & 1.00 & 1.00 \\
$C$ & 1.00 & 2.00 & 5.00 & 5.00 & 1.00 & 1.00 & 1.00 & 1.00 & 1.00 \\
\hline
\end{tabular}

$$
\begin{gathered}
\sum_{j=1}^{n} \tilde{\lambda}_{j} y_{r j} \geq \varphi_{r}^{*} \widetilde{\beta}_{r o}>\varphi_{r}^{*} \bar{\beta}_{r o} \quad r \in O \\
\sum_{j=1}^{n} \tilde{\lambda}_{j} y_{r j} \geq \varphi_{r}^{*} \tilde{\beta}_{r o} \geq \varphi_{r}^{*} \bar{\beta}_{r o} \quad r \notin O \\
\tilde{\lambda}_{j} \geq 0, \quad j=1, \ldots, n .
\end{gathered}
$$

Based on inequalities (53) and (56) there exists $\mu_{i}<1$ for $i \in I$ and $\eta_{r}>1$ for $r \in O$, such that

$$
\begin{gathered}
\sum_{j=1}^{n} \tilde{\lambda}_{j} x_{i j} \leq\left(\mu_{i} \theta_{i}^{*}\right) \bar{\alpha}_{i o}, \quad i \in I \\
\sum_{j=1}^{n} \tilde{\lambda}_{j} y_{r j} \geq\left(\eta_{r} \varphi_{r}^{*}\right) \bar{\beta}_{r o}, \quad r \in O .
\end{gathered}
$$

For each $i=1, \ldots, n$ and $r=1, \ldots, s$, define

$$
\begin{gathered}
\bar{\theta}_{i}= \begin{cases}\mu_{i} \theta_{i}^{*}, & \text { if } i \in I, \\
\theta_{i}^{*}, & \text { if } i \notin I,\end{cases} \\
\bar{\varphi}_{r}= \begin{cases}\eta_{r} \varphi_{r}^{*}, & \text { if } r \in O, \\
\varphi_{r}^{*}, & \text { if } r \notin O .\end{cases}
\end{gathered}
$$

By (54) and (56)-(59), $(\widetilde{\lambda}, \bar{\theta}, \bar{\varphi})$ is a feasible solution of problem (1) (only restrictions on the right have replaced the $X_{o}$ and $Y_{o}$ by $\bar{\alpha}_{o}$ and $\bar{\beta}_{o}$ in problem (1)) where $\bar{\theta}=\left(\bar{\theta}_{1}, \ldots, \bar{\theta}_{m}\right)$ and $\bar{\varphi}=\left(\bar{\varphi}_{1}, \ldots, \bar{\varphi}_{s}\right)$. Therefore

$$
\bar{\rho}_{o}=\frac{(1 / m) \sum_{i=1}^{m} \bar{\theta}_{i}}{(1 / s) \sum_{r=1}^{s} \bar{\varphi}_{r}}<\frac{(1 / m) \sum_{i=1}^{m} \theta_{i}^{*}}{(1 / s) \sum_{r=1}^{s} \varphi_{r}^{*}}=\rho_{o}^{*}
$$

which is against the assumption that is $\operatorname{eff}\left(\bar{\alpha}_{o}, \bar{\beta}_{o}\right)=$ $\operatorname{eff}\left(X_{o}, Y_{o}\right)=\rho_{o}^{*}$.

\section{Illustrative Examples}

Example 1. Consider a problem of three DMU with two inputs $x_{1}, x_{2}$ and two outputs $y_{1}, y_{2}$. The data of inputs, outputs, and ERM-efficiency score are shown in Table 1.

As can be seen, $\mathrm{DMU}_{C}$ is an ERM-efficient DMU. If the decision maker is interested in increasing the output vector from $Y_{C}=(5,5)$ to $\beta_{C}=(6,6)$, then, to solve the model $(5)$ by employing the weight-sum method [25], a Pareto solution for this MOLP is generated as $\alpha_{C}^{*}=(1.20,2.40)$. Based on the model $(6), K_{C}^{*}=1, \operatorname{eff}\left(X_{C}, Y_{C}\right)=\operatorname{eff}\left(\alpha_{C}^{*}, \beta_{C}\right)=1$, which means that the ERM-efficiency remains unchanged. Hence, due to Theorem 6, when the outputs of $\mathrm{DMU}_{A}$ increase to 
TABle 2: The data in Example 2.

\begin{tabular}{lccccc}
\hline DMUs & $x$ & $y$ & $\theta^{*}$ & $\varphi^{*}$ & ERM efficiency \\
\hline$A$ & 1.00 & 1.00 & 0.50 & 1.00 & 0.50 \\
$B$ & 2.00 & 4.00 & 1.00 & 1.00 & 1.00 \\
$C$ & 5.00 & 5.00 & 0.67 & 1.33 & 0.50 \\
$D$ & 6.00 & 7.00 & 0.79 & 1.35 & 0.58 \\
\hline
\end{tabular}

$\beta_{C}=(6,6)$ if the decision maker would like to preserve the efficiency score of this DMU, the inputs should increase to $\alpha_{C}^{*}=(1.20,2.40)$.

Also, if the decision maker is interested in increasing the output vector of $Y_{C}=(5,5)$ to $\beta_{C}=(5.5,6)$, then to solve the model (5) by employing the weight-sum method [25], must the input vector of the $X_{C}=(1,2)$ increase to $\alpha_{C}^{*}=(1.20,2.40)$. Based on model (6), since $K_{C}^{*}=11.5 / 5>$ 1 and $k_{C}^{*}=(6 / 5,1)$, we have $\operatorname{eff}\left(X_{C}, Y_{C}\right) \neq \operatorname{eff}\left(\alpha_{C}^{*}, \beta_{C}\right)=$ 0.96; therefore, according to part (ii) of Theorem 6, we have $\operatorname{eff}\left(X_{C}, Y_{C}\right)=\operatorname{eff}(1.20,2.40,6 / 5 * 5.5,1 * 6)=1$. This indicates that new DMU in output component the first to amount " 0.5 " of the lack produce. In other words, using model (4), we have $\operatorname{eff}\left(X_{C}, Y_{C}\right)=\operatorname{eff}(1.20,2.40,6 / 5 * 5.5,1 * 6)=1$.

Example 2. Consider a problem of four DMU with one input $x$ and one output $y$. The data of input, output, and ERMefficiency scores are shown in Table 2.

As can be seen, $\mathrm{DMU}_{B}$ is an ERM-efficient DMU. Assume the decision maker identified rate of increase input and output for this DMU, respectively, as $2 \leq x \leq 7,4 \leq y \leq 10$. In order to propose patterns to the decision maker to increase input and output for this DMU, under preserving the ERMefficiency index, based on model (49) the following MOLP model is considered:

$$
\begin{array}{ll}
\min & \alpha_{B} \\
\max & \beta_{B} \\
\text { s.t. } & \lambda_{1}+2 \lambda_{2}+5 \lambda_{3}+6 \lambda_{4} \leq \theta_{B}^{*} \alpha_{B} \\
& \lambda_{1}+4 \lambda_{2}+5 \lambda_{3}+7 \lambda_{4} \geq \varphi_{B}^{*} \beta_{B} \\
& \alpha_{B} \geq 2, \quad \beta_{B} \geq 4 \\
& \alpha_{B} \leq 7, \quad \beta_{B} \leq 10 \\
& \lambda_{j} \geq 0, \quad j=1, \ldots, 4 .
\end{array}
$$

Using the weight-sum method [25], a Pareto solution is generated for this MOLP as $\left(\alpha_{B}^{*}, \beta_{B}^{*}\right)=(5,10)$. Therefore, according to Theorem 15, when the inputs and outputs of $\mathrm{DMU}_{B}$ increase to 5 and 10 , respectively, then the ERMefficiency score of this DMU is 1.

\section{Conclusion}

In the present paper, a typical inverse optimization problem on the nonradial enhanced Russell model has been studied: two main questions on inverse DEA have been discussed and the models have been proposed to estimate the input (output) levels of a given DMU when some or all its output (input) levels were increased under the constant ERM-efficiency score. To determine sufficient and necessary conditions of estimated inputs (outputs), Pareto solutions of the MOLP were used. Moreover, in finding inputs (outputs), if there exists lack output (extra input) in each of a output (input) components, the amount of the lack (extra) is specified by auxiliary models. For an ERM-efficient DMU, necessary and sufficient conditions were introduced to find the minimum and maximum increase of input and output levels, respectively, provided that the ERM-efficiency score remains unchanged. Therefore, the patterns can be presented to the decision maker in order to increase inputs and outputs (extending decision making units) for an ERM-efficient DMU such that the ERM-efficiency remains unchanged. In other words, inverse DEA can be used in theoretical and practical purposes such as strategic planning, management control, resource allocation, and ranking. The sufficient conditions were established only for the ERM-efficient DMU, but the given necessary conditions were for each ERM-efficient or ERM-inefficient DMU. Finding sufficient conditions for ERM-inefficient $\mathrm{DMU}_{s}$ can be a suitable research field. In addition, using nonradial models under intertemporal dependence, solving the problems introduced by Wei et al. [14], Hadi-Vencheh et al. [16], and the new problem discussed in this paper can be a useful research topic.

\section{Conflict of Interests}

The authors declare that there is no conflict of interests regarding the publication of this paper.

\section{References}

[1] A. Charnes, W. W. Cooper, and E. Rhodes, "Measuring the efficiency of decision making units," European Journal of Operational Research, vol. 2, no. 6, pp. 429-444, 1978.

[2] R. D. Banker, A. Charnes, and W. W. Cooper, "Some models for estimating technical and scale efficiencies in data envelopment analysis," Management Science, vol. 30, no. 9, pp. 1078-1092, 1984.

[3] W. D. Cook and L. M. Seiford, "Data envelopment analysis (DEA)-thirty years on," European Journal of Operational Research, vol. 192, no. 1, pp. 1-17, 2009.

[4] W. W. Cooper, L. M. Seiford, and K. Tone, Data Envelopment Analysis: A Comprehensive Text With Models, Applications, References and DEA-Solver Software, Kluwer Academic, New York, NY, USA, 1999.

[5] A. Hatami-Marbini, A. Emrouznejad, and M. Tavana, "A taxonomy and review of the fuzzy data envelopment analysis literature: two decades in the making," European Journal of Operational Research, vol. 214, pp. 457-472, 2011.

[6] B. Golany, "An interactive MOLP procedure for the extension of DEA to effectiveness analysis," Journal of the Operational Research Society, vol. 39, pp. 725-734, 1988.

[7] F. Hosseinzadeh Lotfi, G. R. Jahanshahloo, A. Ebrahimnejad, M. Soltanifar, and S. M. Mansourzadeh, "Target setting in the general combined-oriented CCR model using an interactive MOLP method," Journal of Computational and Applied Mathematics, vol. 234, no. 1, pp. 1-9, 2010. 
[8] F. H. Lotfi, G. R. Jahanshahloo, M. Soltanifar, A. Ebrahimnejad, and S. M. Mansourzadeh, "Relationship between MOLP and DEA based on output-orientated CCR dual model," Expert Systems with Applications, vol. 37, no. 6, pp. 4331-4336, 2010.

[9] T. Joro, P. Korhonen, and S. Zionts, "An interactive approach to improve estimates of value efficiency in data envelopment analysis," European Journal of Operational Research, vol. 149, no. 3, pp. 688-699, 2003.

[10] E. Thanassoulis and R. Allen, "Simulating weights restrictions in data envelopment analysis by means of unobserved DMUs," Management Science, vol. 44, no. 4, pp. 586-594, 1998.

[11] B. Y. H. Wong, M. Luque, and J. B. Yang, "Using interactive multiobjective methods to solve DEA problems with value judgements," Computers and Operations Research, vol. 36, no. 2, pp. 623-636, 2009.

[12] J. B. Yang, B. Y. H. Wong, D. L. Xu, and T. J. Stewart, "Integrating DEA-oriented performance assessment and target setting using interactive MOLP methods," European Journal of Operational Research, vol. 195, no. 1, pp. 205-222, 2009.

[13] X. S. Zhang and J. C. Cui, "A project evaluation system in the state economic information system of china an operations research practice in public sectors," International Transactions in Operational Research, vol. 6, pp. 441-452, 1999.

[14] Q. L. Wei, J. Zhang, and X. Zhang, "Inverse DEA model for inputs/outputs estimate," European Journal of Operational Research, vol. 121, no. 1, pp. 151-163, 2000.

[15] H. Yan, Q. Wei, and G. Hao, "DEA models for resource reallocation and production input/output estimation," European Journal of Operational Research, vol. 136, no. 1, pp. 19-31, 2002.

[16] A. Hadi-Vencheh, A. A. Foroughi, and M. Soleimani-damaneh, "A DEA model for resource allocation," Economic Modelling, vol. 25, no. 5, pp. 983-993, 2008.

[17] A. Hadi-Vencheh and A. Asghar Foroughi, "A generalized DEA model for inputs/outputs estimation," Mathematical and Computer Modelling, vol. 43, no. 5-6, pp. 447-457, 2006.

[18] G. R. Jahanshahloo, F. H. Lotfi, N. Shoja, G. Tohidi, and S. Razavyan, "Input estimation and identification of extra inputs in inverse DEA models," Applied Mathematics and Computation, vol. 156, no. 2, pp. 427-437, 2004.

[19] G. R. Jahanshahloo, F. Hosseinzadeh Lotfi, N. Shoja, G. Tohidi, and S. Razavyan, "The outputs estimation of a DMU according to improvement of its efficiency," Applied Mathematics and Computation, vol. 147, no. 2, pp. 409-413, 2004.

[20] S. Lertworasirikul, P. Charnsethikul, and S.-C. Fang, "Inverse data envelopment analysis model to preserve relative efficiency values: The case of variable returns to scale," Computers and Industrial Engineering, vol. 61, no. 4, pp. 1017-1023, 2011.

[21] H. T. Lin, "An efficiency-driven approach for setting revenue target," Decision Support Systems, vol. 49, no. 3, pp. 311-317, 2010.

[22] L. M. Seiford and R. M. Thrall, "Recent developments in DEA. The mathematical programming approach to frontier analysis," Journal of Econometrics, vol. 46, no. 1-2, pp. 7-38, 1990.

[23] R. Färe and S. Grosskopf, "A nonparametric cost approach to scale efficiency," Scandinavian Journal of Economics, vol. 87, pp. 594-604, 1985.

[24] J. T. Pastor, J. L. Ruiz, and I. Sirvent, "An enhanced DEA Russell graph efficiency measure," European Journal of Operational Research, vol. 115, pp. 596-607, 1999.

[25] M. Ehrgott, Multicriteria Optimization, Springer, Berlin, Germany, 2005. 


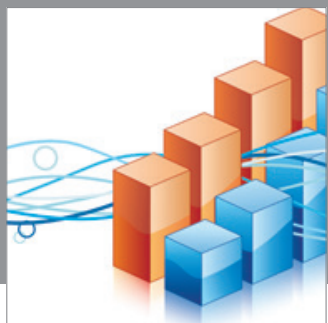

Advances in

Operations Research

mansans

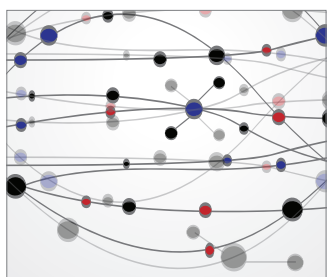

The Scientific World Journal
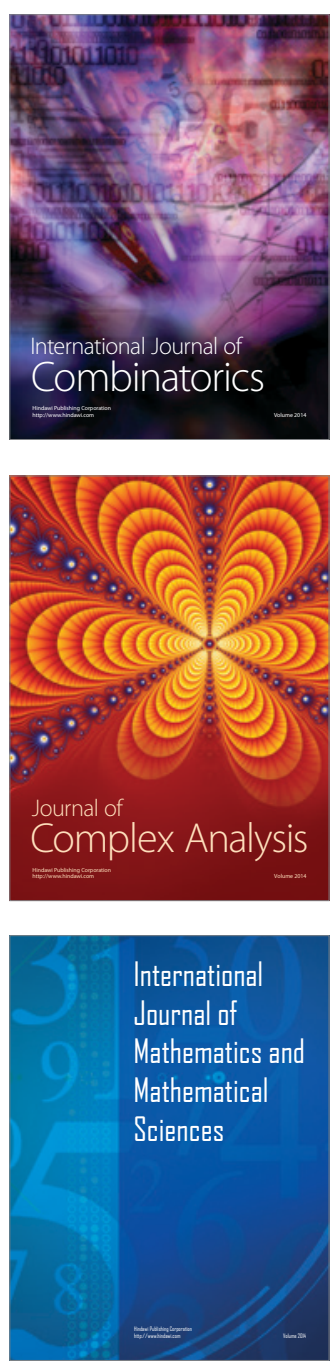
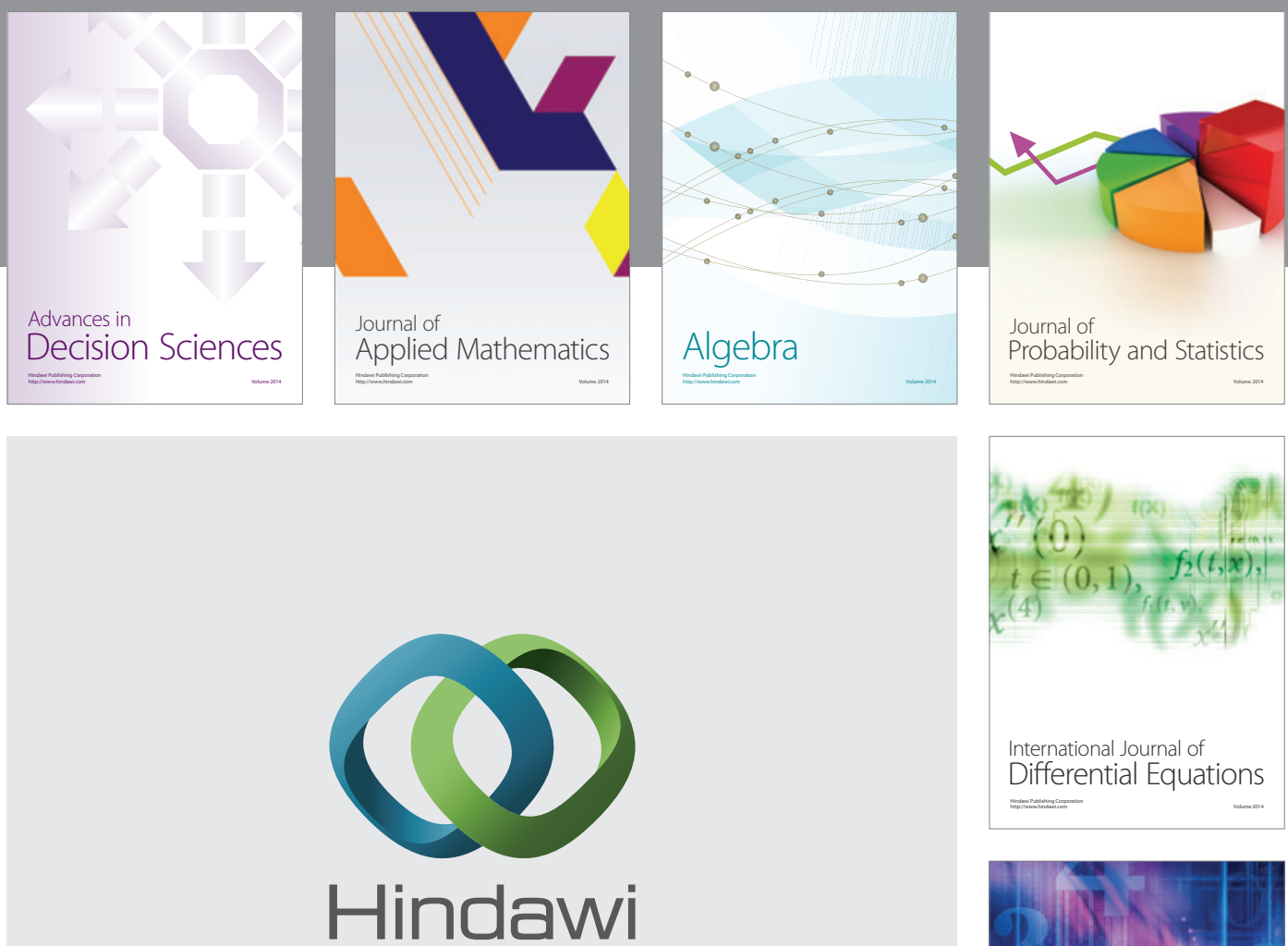

Submit your manuscripts at http://www.hindawi.com
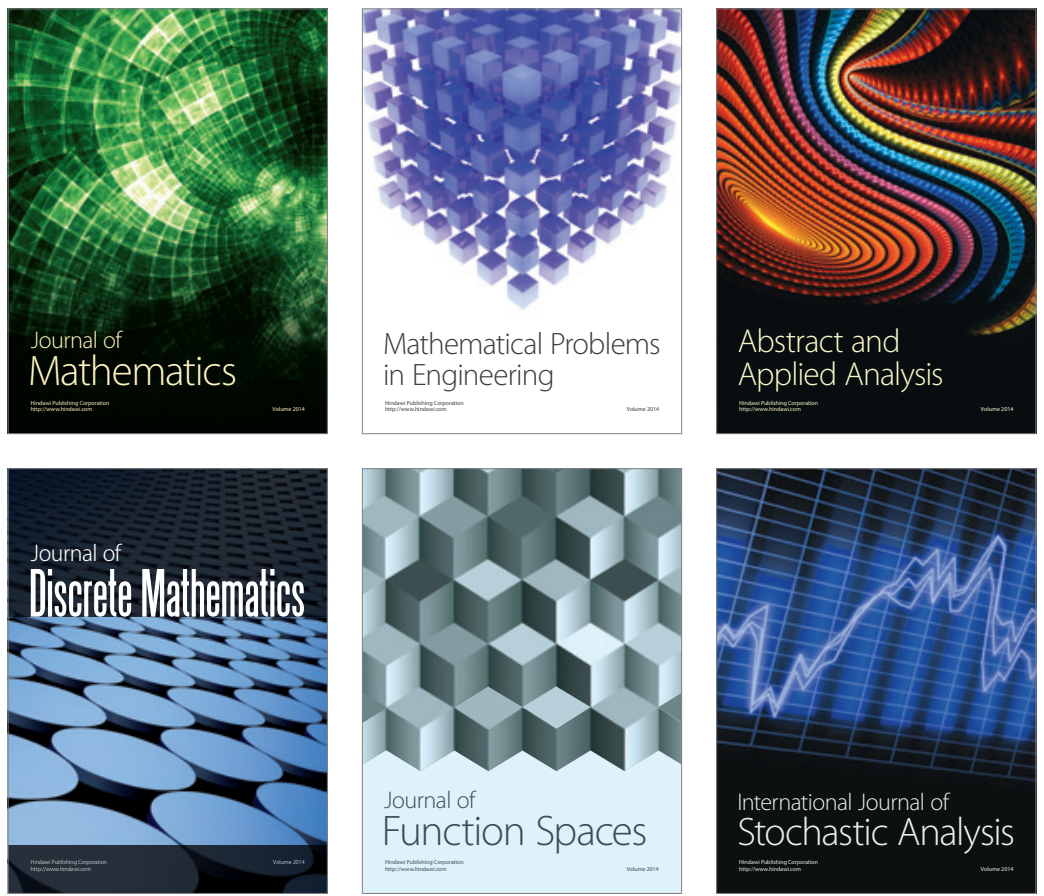

Journal of

Function Spaces

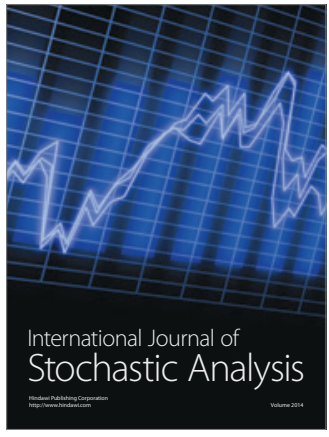

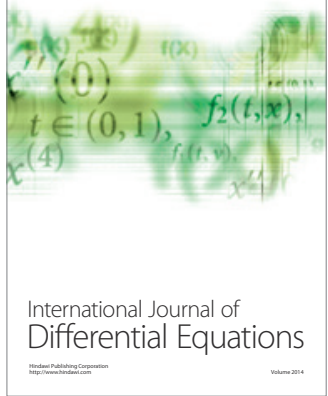
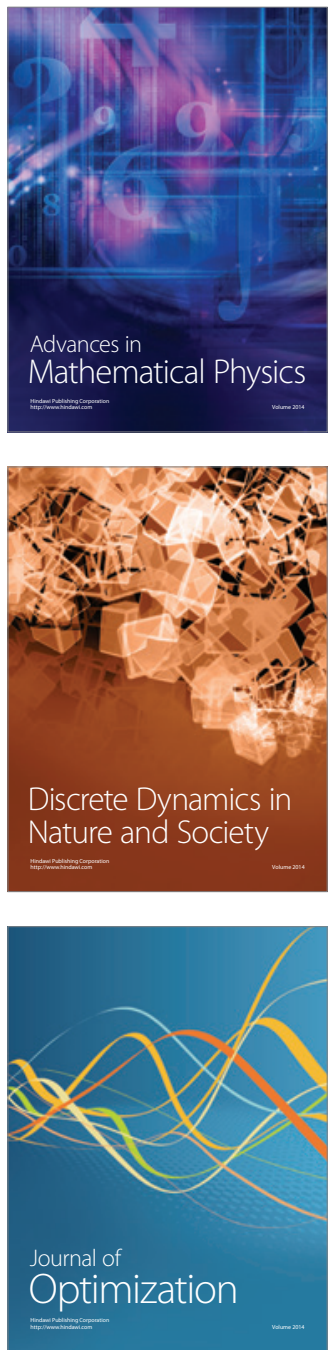